\section{IDDF2019-ABS-0060 THE DIFFERENCE BETWEEN BRUSEIN-D LIGAND FROM MAKASSAR ETHANOLIC FRACTION (BRUCEA JAVANICA (L.) MERR) AND GEMCITABINE ON THE INHIBITION OF PANC-1 PANCREAS CARCINOMA GROWTH AND MUTANT P53 EXPRESSION}

${ }^{1}$ Budhi Ida Bagus*, ${ }^{2}$ Hernanto Jody, ${ }^{3}$ Metria Ida Bagus, ${ }^{4}$ Setyawati Ida Ayu. ${ }^{1}$ Department of Surgery, Sebelas Maret University, Indonesia; ${ }^{2}$ Department of Surgery, Moewardi General Hospital, Indonesia; ${ }^{3}$ Medical Faculty, Sebelas Maret University, Indonesia; ${ }^{4}$ Medical Faculty, Pendidikan Ganesha University, Indonesia

\subsection{6/gutjnl-2019-IDDFabstracts.20}

Background Giving Brusein D or Gemcitabine to pancreatic carcinoma cells will re-infect genes that act as tumor suppressor genes. In Brusein D administration there will be activation of p38-MAPK, caspase 9 and caspase 3 as well as inhibition of NF-kB which will stop the synthesis of pancreatic carcinoma DNA so that the cell will carry out cell degradation by apoptosis. In the administration of Gemcitabine, there will be tumor suppressor gene activation, p38-MAPK, which in turn will trigger cell degradation with the mechanism of apoptosis.

Methods PANC-1 pancreatic carcinoma cell cultures were divided into three groups. The first group was in control, the second group was treated with three repetitions of the dose of gemcitabine, the third group was treated with three repetitions of the Brusein D dose, then the number of mutant p53 was calculated in each group.

Results Mutant p53 expression levels in cultures of pancreatic carcinoma PANC-1 cells in vitro were the least in the Brusein D treatment $2 \mathrm{ug} / \mathrm{ml}$ (B 2), which average 12.7\%, then Gemcitabine $2 \mathrm{ug} / \mathrm{ml}$ (G 2) ) which is an average of $16.7 \%$, followed by Brusein D $1 \mathrm{ug} / \mathrm{ml}$ (B 1) which is an average of $22.0 \%$, then followed by Gemcitabine $1 \mathrm{ug} / \mathrm{ml}$ (G 1) which is an average of $22.2 \%$, followed by Brusein D $0.5 \mathrm{ug} / \mathrm{ml}$ (B 0.5 ) which is an average of $28.8 \%$, then followed by Gemcita-

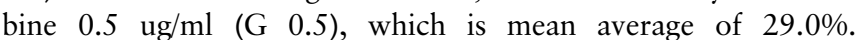
And the highest level of mutant p53 expression was control, with an average of $51.3 \%$.

Conclusions Brusein D ligand from an ethanolic fraction of Makassar Fruit (Brucea javanica (L.) Merr) has the inhibitory potential for mutant p53 expression so that it can be a candidate for the chemotherapeutic agent in cancer with mutations through the p53 pathway.

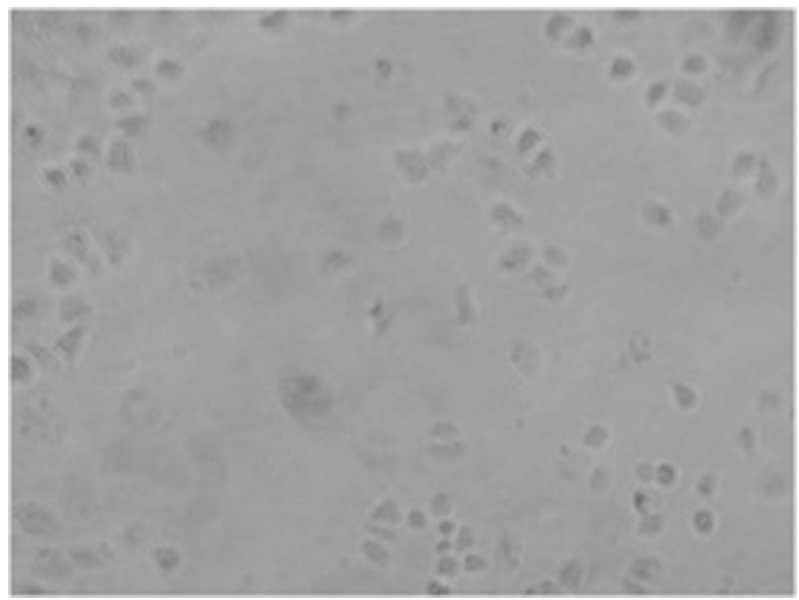

Abstract IDDF2019-ABS-0060 Figure 1 Brusein D inhibition on panc 1 pancreas carcinoma

\section{IDDF2019-ABS-0067 INCOMPLETE RADIOFREQUENCY ABLATION OF HCC PROMOTES INTRAHEPATIC METASTASIS AND STEMNESS IN A VEGFR1-DEPENDENT MANNER}

Manling Huang*, Sui Peng. Department of Gastroenterology, First Affiliated Hospital, Sun Yat-sen University, China

\subsection{6/gutjnl-2019-IDDFabstracts.21}

Background Approximately 50\% hepatocellular carcinoma (HCC) patients recur within three years after radiofrequency ablation (RFA). Incomplete RFA of HCC could activate HIF/ VEGF pathway to mediate malignant transition, and VEGF signaling was reported to enhance cancer stemness in skin cancer and Glioblastoma multiforme. We aimed to investigate the role of VEGF signaling on the process of incomplete RFArelated intrahepatic metastasis and stemness enhancement in HCC.

Methods HCC patient-derived xenograft (PDX) mice model and sublethal heat treatment cell model were established to mimic clinical setting of incomplete RFA. Cancer stem cell markers, MMPs, VEGF pathway, proliferation, migration and sphere were evaluated.

Results The expression of cancer stem cell (CSC) markers CD133, CD44, EpCAM was increased in tumor tissues with incomplete RFA from PDX mice compared to tumor tissues before RFA. Metastatic markers MMP2, MMP7 and MMP9 were also upregulated in tumor tissues with incomplete RFA, with increasing VEGFR1 and decreasing VEGFR2 expression. After sublethal heat treatment, enhanced cell migration ability was observed in HepG2, HCCLM3 and SMMC7721 cells, which coincided with an enhanced ability of sphere formation and upregulation of VEGFR1, CD133, CD44, and EpCAM. Further exploration revealed that HCC cells secreted more VEGF after heat-treatment. VEGF promoted the stemness and migration of HCC cells, which could not be suppressed by inhibiting VEGFR2. On the other hand, PIGF, the ligand of VEGFR1, significantly enhanced the stemness and migration of HCC cells. More importantly, blocking VEGFR1 reduced heat-induced stemness and migration, while inhibition of VEGFR2 did not reduce the stemness and migration of HCC cells with heat treatment.

Conclusions Sublethal heat treatment could increase the migration capability and stem cell-like phenotype of HCC cells. VEGFR1 played a critical role in such malignant transition after sublethal heat treatment, suggesting VEGFR1 may serve as a potential and promising therapeutic target for preventing recurrence after RFA.

\section{IDDF2019-ABS-0069 REDUCED EXPRESSION OF CIRCRNA HSA_CIRC_001888 IN GASTRIC CANCER AND ITS CLINICAL SIGNIFICANCE}

${ }^{1}$ Peina Shi* ${ }^{2}$ Haojun Song, ${ }^{2}$ Xiaoyun Ding. ${ }^{1}$ The Medical School of Ningbo University, China; ${ }^{2}$ The Gastroenterology Department of Ningbo First Hospital, China

\subsection{6/gutjnl-2019-IDDFabstracts.22}

Background Circular RNAs (circRNAs) are a new class of endogenous noncoding RNAs. Owing to the special covalently closed loop structure without 5' end caps or 3' poly (A) tails, circRNAs are more stable and conserved than linear RNAs. Growing evidence shows that circRNAs play a crucial role in the occurrence and development of several human diseases, 

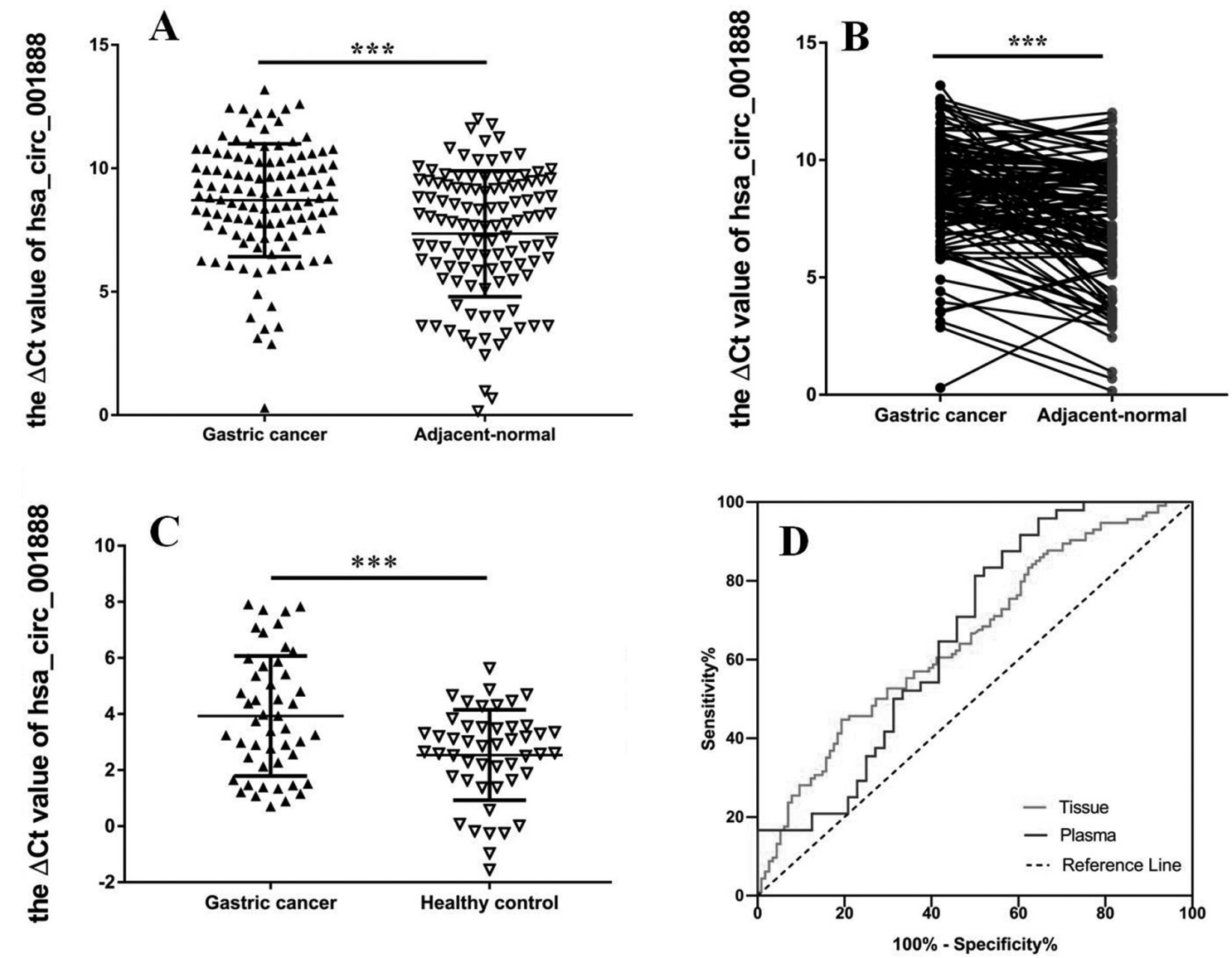

\section{Abstract IDDF2019-ABS-0069 Figure 1}

especially cancers. However, little is known about their expression and function in gastric cancer (GC).

Methods The hsa_circ_001888 levels in 114 paired GC tissues and adjacent non-tumor tissues, 48 plasma samples from patients with GC and 48 plasma samples from health controls were detected by quantitative reverse transcription-polymerase chain reaction (qRT-PCR). Then, the relationships between hsa_circ_001888 expression and the clinicopathological features of patients with GC were further analyzed. Finally, a receiver operating characteristic (ROC) curve was generated to evaluate the diagnostic value of hsa circ 001888 .

Results Hsa_circ_001888 was first found to be significantly down-regulated in GC tissues $(\mathrm{p}<0.001$, (figure $1 \mathrm{~A}, \mathrm{~B})$ ) and plasma samples from patients with GC $(\mathrm{p}<0.001$, (figure 1C). The higher $\Delta \mathrm{Ct}$ value indicates lower expression. Moreover, we analyzed their association with clinicopathological features of patients with GC. Clinicopathological features showed that hsa_circ_001888 level in GC tissues was correlated with differentiation and in GC plasma linked with CEA and CA199 expression. We did not further find any association between its levels with other clinicopathological features such as age, gender, invasion, lymphatic metastasis. To evaluate the potential diagnostic value, the ROC curve was used. The area under the ROC curve of has_circ_001888 in tissues and plasma were up to 0.654 and $0 . \overline{6} 62$, respectively (figure $1 \mathrm{D}$ ), suggesting good diagnostic value.

Conclusions These results indicated that hsa_circ_001888 was significantly down-regulated in GC and may serve as a novel potential biomarker in the diagnosis of GC.

\section{IDDF2019-ABS-0080 ROSIGLITAZONE ALLEVIATES LPS-INDUCED INFLAMMATION IN RAW264.7 CELLS VIA THE INHIBITION OF NF- $K B$ IN A PPAR $\gamma$ - DEPENDENT MANNER}

Jingping Zhou*, Xiaoning Yang, Yang Song, Yiqun Hu. Department of Gastroenterology, Zhongshan Hospital Affiliated to Xiamen University, China

\subsection{6/gutjnl-2019-IDDFabstracts.23}

Background Rosiglitazone is a synthetic peroxisome proliferator-activated receptor $\gamma(\operatorname{PPAR} \gamma)$ agonist that is widely used to treat type 2 diabetes, recent research has highlighted its antiinflammatory activity. The aim of this study was to investigate whether rosiglitazone can alleviate the decline in RAW264.7cells viability due to lipopolysaccharide(LPS) induced inflammation and the underlying mechanism.

Methods RAW264.7 macrophages were stimulated with 100 $\mathrm{ng} / \mathrm{ml}$ LPS to establish an inflammatory injury model. Cells were treated with LPS and various concentrations of rosiglitazone. Cell viability was assessed by MTT assays. Inflammatory cytokines were detected by ELISA and qRT-PCR.Nitric Oxide (NO) production was accessed using the Griess reagent system. The expression levels of key proteins in the NF- $\mathrm{BB}$ pathway were detected by western blotting.

Results Rosiglitazone alleviated the decline in RAW264.7 cells viability induced by LPS and inhibited inflammatory cytokine expression in a concentration-dependent manner. Rosiglitazone significantly inhibited the upregulation of p65 phosphorylation and the downregulation of I $\mathrm{B} \alpha$ induced by LPS. The inhibitory effects could be blocked by PPAR $\gamma$ knockdown. 